Rev. Bras. Saúde Prod. Anim., Salvador, v.16, n.1, p.199-209 jan./mar., 2015 http://www.rbspa.ufba.br

\title{
Parâmetros fisiológicos e desempenho para ovelhas Santa Inês e cordeiros 1/2 Dorper - Santa Inês nas estações verão e inverno
}

\section{Physiological parameters and performance of Santa Inês sheep and Dorper lambs 1/2 - Santa Ines in summer and winter season}

\author{
QUEIROZ, Edicarlos Oliveira ${ }^{1}$; MACEDO, Francisco de Assis Fonseca de ${ }^{2}$; \\ BARBOSA, Orlando Rus ${ }^{2}$; ZANCANELA, Vittor ${ }^{2}$; MORA, Natália Holtz Alves \\ Pedroso $^{2}$; BALISCEI, Márcio Antonio ${ }^{3}$
}

\footnotetext{
${ }^{1}$ Universidade Federal do Mato Grosso, Departamento de Zootecnia, Rondonópolis, Mato Grosso, Brasil.

${ }^{2}$ Universidade Estadual de Maringá, Departamento de Zootecnia, Maringá, Paraná, Brasil.

${ }^{3}$ Emater, Terra Roxa, Paraná, Brasil.

*Endereço para correspondência: edqueiroz@ hotmail.com
}

\section{RESUMO}

Objetivou-se avaliar os parâmetros fisiológicos e o desempenho de ovelhas da raça Santa Inês e cordeiros $1 / 2$ Dorper - Santa Inês nas estações verão e inverno. Foram utilizadas 160 ovelhas da raça Santa Inês e 189 cordeiros 1/2 Dorper Santa Inês em delineamento inteiramente casualizado distribuídas em dois tratamentos, verão: 80 ovelhas e 92 cordeiros e no inverno: 80 ovelhas e 97 cordeiros. Registrou-se os parâmetros ambientais, temperatura do ar $\left(\mathrm{Ta}^{\circ} \mathrm{C}\right)$, umidade relativa (UR\%), velocidade do vento $\left(\mathrm{Vv} \mathrm{m} \cdot \mathrm{s}^{-1}\right)$ e temperatura do globo negro $\left(\mathrm{Tg}^{\circ} \mathrm{C}\right)$ e parâmetros fisiológicos, temperatura retal $\left(\mathrm{TR}^{\circ} \mathrm{C}\right)$, frequência respiratória $(\mathrm{FR}$ mov. min $^{-1}$ ) e frequência cardíaca (FC bat. $\min ^{-1}$ ). A condição corporal das ovelhas foi aferida ao parto. No terço final de gestação verificou-se efeito significativo $(\mathrm{P}<0,05)$ para TR, FR e FC nas ovelhas, em que no verão os valores foram, respectivamente, $40,63^{\circ} \mathrm{C}, 102,58\left(\mathrm{mov} \cdot \mathrm{min}^{-1}\right) \mathrm{e}$ 128,34 (bat. $\mathrm{min}^{-1}$ ) com ICT 46, e no inverno foi de $38,34^{\circ} \mathrm{C}, 35,71\left(\right.$ mov. $\left.^{-1}{ }^{-1}\right), 77,96\left(\right.$ bat. $^{-1}{ }^{-1}$ ) com ICT de 14. Os valores de TR, FR e FC ao parto e ao desmame também foram significativas entre as estações verão e inverno. Foram verificadas diferenças $(\mathrm{P}<0,05)$ para a TR, FR e FC dos cordeiros ao nascimento e ao desmame entre o verão e inverno. $\mathrm{O}$ peso ao nascer $(4,51 \pm 2,86)$ e ao desmame $(16,91 \pm 3,91)$ foram superiores para os cordeiros nascidos no verão. Durante o verão, ovelhas da raça Santa Inês foram superiores em escore de condição corporal, favorecendo nascimentos de cordeiros mais pesados e consequentemente peso ao desmame superior, com taxa de sobrevivência acima da encontrada no inverno.

Palavras-chave: batimentos cardíacos, bioclimatologia, frequência respiratória, temperatura corporal

\section{SUMMARY}

This study aimed to evaluate the physiological parameters and performance of sheep Santa Inês and Dorperlambs $1 / 2$-SantaInes in summer and winter seasons. 160 Santa Inês sheep and 189 1/2 Dorper lambs were used - Santa Inês completely randomized in two treatments, summer: 80 sheep and 92 lambs and winter: 80 sheep and 97 lambs.Was recorded environmental the environmental parameters of air temperature $\left(\mathrm{Ta}^{\circ} \mathrm{C}\right)$, relative humidity $(\% \mathrm{RH})$, wind speed (Vvms- 1) and black globe temperature $\left(\mathrm{Tg}^{\circ} \mathrm{C}\right)$ and physiological parameters of rectal temperature $\left(\operatorname{Tr}^{\circ} \mathrm{C}\right)$, respiratory rate $(\mathrm{RR}$ mov.min-1) and heart rate (HR beat.min - 1). The body condition of the ewes was measured at birth. In the final third of gestation there was a significant effect $P<0.05$ ) for TR, FR and HR on sheep, where in summer the values were $40.63^{\circ} \mathrm{C}, \quad 102.58$ (mov.min-1) and 128,34 (beat.min-1) with $46 \mathrm{ICT}$, and winter was $38.34^{\circ} \mathrm{C}$, 35.71 (mov.min-1), 77.96 (beat.min1) with ICT of 14. The values of TR, RR and $\mathrm{HR}$ at birth and weaning were also significant between the summer and winter seasons. Differences were observed $(\mathrm{P}<0.05)$ for $\mathrm{TR}$, $\mathrm{RR}$ and $\mathrm{HR}$ of lambs at birth and weaning 
between summer and winter. The weight at birth $(4.51 \pm 2.86)$ and weaning $(16.91 \pm 3.91)$ were higher for those born in the summer. During the summer Santa Inês ewes were superior in body condition score, favoring births of heavier lambs and consequently higher weaning weight with higher rate of survival than those found in winter.

Keywords: bioclimatology, body temperature, heartbeats, respiratory Rate

\section{INTRODUÇÃO}

O clima é um fator determinante na produção animal, principalmente pela interação animal x ambiente. Segundo Neiva et al. (2004), esta interação deve ser considerada para se buscar maior eficiência na produção animal. O clima pode interagir com os animais, alterando suas respostas fisiológicas, comportamentais e produtivas.

A sobrevivência dos mamíferos ou mesmo a sua produtividade dependem principalmente de sua capacidade em manter a temperatura corporal dentro de limites da temperatura crítica inferior (TCI) e temperatura crítica superior (TCS), processo este denominado de homeotermia. A zona de conforto térmico para ovinos está na faixa de -2 a $20^{\circ} \mathrm{C}$ (RUCKEBUSH et al., 1991). Critérios de tolerância e adaptação dos animais são determinados pelas medidas fisiológicas da respiração, batimentos cardíacos e temperatura corporal. Neiva et al. (2004) descreveram o efeito do estresse climático sobre os parâmetros fisiológicos, consumo de matéria seca e desempenho de ovinos da raça Santa Inês, em que a elevação da temperatura ambiente desprotegido exerceu influência sobre a temperatura retal e frequência respiratória. Os animais mantidos á sombra apresentaram ganho de peso (174g/dia) aproximadamente $30 \%$ maior que aqueles mantidos ao sol (122g/dia), destacando a importância da avaliação do ambiente para se alcançar boa produtividade animal.

A avaliação dos parâmetros fisiológicos dos cordeiros do nascimento á desmama é uma medida importante para identificar os pontos críticos da variação do ambiente sobre o estresse dos animais, conforme Nowak \& Poindron (2006) nos primeiros 15 minutos de vida do cordeiro, a temperatura interna diminuiu entre 1 a $2^{\circ} \mathrm{C}$ em relação à temperatura intrauterina de $39^{\circ} \mathrm{C}$.

Dada a susceptibilidade dos neonatos às intempéries, especialmente sob condições extensivas, diversos autores recomendam formas de controle ambiental e conforto aos animais como posicionamento estratégico de sombra, abrigos, quebraventos, aquecedores, alimento, e água nos piquetes destinados às ovelhas recémparidas e cordeiros (BLACKSHAW, 2003; CORNER et al., 2006).

O rebanho materno poliéstrico não estacional, como a raça Santa Inês, possibilita a programação do nascimento de cordeiros durante todo ano. Essa metodologia é de fundamental importância para consolidação da cadeia de carne de cordeiro. Dessa forma, tornase necessário avaliar as respostas fisiológicas e produtivas de ovelhas da raça Santa Inês e cordeiros 1/2 Dorper Santa Inês na estação de verão e inverno.

\section{MATERIAL E MÉTODOS}

O trabalho foi realizado na propriedade Bela Cabanha, situada no distrito de Maristela, município de Alto Paraná Paraná - Brasil. O clima, segundo a classificação de Köppen, é subtropical, a temperatura média é inferior a $18^{\circ} \mathrm{C}$ (mesotérmico) e temperatura média superior é acima de $22^{\circ} \mathrm{C}$, com verões quentes, geadas pouco frequentes $\mathrm{e}$ tendência de concentrações das chuvas 
nos meses de verão; contudo, sem estação seca definida.

O período experimental foi dividido em duas etapas, segundo as estações do ano, definidas para uma região de clima tropical, verão e inverno. Procedeu-se o acompanhamento de quatro períodos de parição, dois períodos no verão e dois no inverno. A primeira coleta ocorreu de dezembro de 2010 a março de 2011; a segunda de maio a agosto de 2011; a terceirade dezembro de 2011 a março de 2012 e a quarta de maio a agosto de 2012.

Utilizaram-se 160 ovelhas multíparas da raça Santa Inês com pelagem de coloração preta, divididas em dois tratamentos inteiramente casualizados: verão (V) $\mathrm{n}=80$ ovelhas e seus respectivos cordeiros $1 / 2$ Dorper - Santa Inês, $\mathrm{n}=92$. No tratamento inverno (I) $\mathrm{n}=80$ ovelhas e os cordeiros nascidos, $\mathrm{n}=97$ cordeiros $1 / 2$ Dorper - Santa Inês, totalizando 189 cordeiros observados. As ovelhas foram mantidas em pastagem de grama Estrela (Cynodonnlemfuensis) e Tifton (Cynodonssp) da cobertura até o terço final de gestação. As avaliações bromatológicas não foram realizadas.

No terço final de gestação, as ovelhas foram conduzidas para um piquete maternidade com a mesma pastagem, sal e água "ad libitum" e abrigo. Após o parto, foram mantidas por sete dias em um galpão maternidade coberto e após este período, ovelhas e cordeiros, voltaram para a pastagem. As ovelhas foram vermifugadas com $1 \mathrm{~mL}$ de Cydectim (moxidectina), para ovelhas com peso até $50 \mathrm{~kg}$, e $2 \mathrm{~mL}$ com peso acima de $50 \mathrm{~kg}$, tendo como referência os resultados do monitoramento obtido por meio do método Famacha®.

Os cordeiros tiveram acesso livre à ração comercial peletizada com $20 \%$ de Proteína Bruta (PB) e $62 \%$ de Nutrientes Digestíveis Totais (NDT), em sistema "creepfeeding", a partir da segunda semana de vida, e mantidos com suas mães até o desmame, aos 60 dias de idade. Os cordeiros tiveram acesso a uma mistura de sal mineral "ad libitum" e receberam vacinas (ectima contagioso, carbúnculo, gangrena gasosa e enterotoxemia).

A sobrevivência e o desempenho dos cordeiros foram realizados no momento do desmame, registrando-se o ganho de peso diário dos cordeiros. $\mathrm{O}$ escore de condição corporal das ovelhas (ECC) registrado ao parto, atribuindo-se notas de 1 a 5 , fracionadas em 0,25 , sendo 1 para ovelhas extremamente magras e 5 para obesas. Para verificação do desempenho dos cordeiros por meio do ECC das ovelhas, agruparam-se ovelhas com mesmo escore e procedeu a avaliação.

Os parâmetros ambientais foram registrados durante o terço final de gestação, ao parto e desmame por meio de termo-higrô-anemômetro $\left(\right.$ THAL300 ${ }^{\circledR}$ ), sendo que as variáveis temperatura do ar e umidade relativa foram coletadas em leitura instantânea e para cada dado da variável velocidade do vento foi utilizada a média entre o valor máximo e o mínimo, ocorrido em 10 segundos de leitura, por ser um parâmetro de grande variação. A temperatura do Globo Negro foi obtida com o uso de uma globo esfera plástica com $15 \mathrm{~cm}$ de diâmetro e termômetro de coluna de álcool. A temperatura do ponto de orvalho e a pressão parcial de vapor foram obtidas através de equações psicométricas, conforme Silva (2000). Para avaliar os parâmetros ambientais, os equipamentos foram posicionados a $0,5 \mathrm{~m}$ de altura do solo, simulando a altura do dorso dos animais. Encontra-se, na Tabela 1, os dados climáticos no período experimental coletados no terço final de gestação, ao parto e no desmame dos cordeiros. 
Rev. Bras. Saúde Prod. Anim., Salvador, v.16, n.1, p.199-209 jan./mar., 2015 http://www.rbspa.ufba.br ISSN 15199940

Tabela 1. Médias de temperatura do ar (Ta), umidade relativa (UR) e velocidade do vento $(\mathrm{Vv})$, no terço final de gestação, ao parto e desmame durante o verão e inverno

\begin{tabular}{lcccccc}
\hline $\begin{array}{l}\text { Momentos de } \\
\text { observação }\end{array}$ & \multicolumn{3}{c}{ Verão } & \multicolumn{3}{c}{ Inverno } \\
\cline { 2 - 6 }$\left({ }^{\circ} \mathrm{C}\right)$ & $\mathrm{UR}(\%)$ & $\mathrm{Vv}\left(\mathrm{m} . \mathrm{s}^{-1}\right)$ & $\mathrm{Ta}\left({ }^{\circ} \mathrm{C}\right)$ & $\mathrm{UR}(\%)$ & $\mathrm{Vv}\left(\mathrm{m} \cdot \mathrm{s}^{-1}\right)$ \\
\hline Terço final & 33,5 & 62,6 & 1,9 & 10,8 & 67,8 & 2,2 \\
Ao parto & 28,4 & 67,7 & 2,0 & 13,7 & 76,2 & 2,2 \\
Desmame & 31,5 & 64,8 & 1,9 & 16,9 & 50,3 & 2,5 \\
\hline
\end{tabular}

Os parâmetros fisiológicos para ovelhas foram coletados durante o terço final de gestação e ao parto, logo após a primeira mamada dos cordeiros para não interferir no vínculo materno-filial e ao desmame. Para os cordeiros, estes parâmetros foram obtidos ao nascer, logo após a primeira mamada para não interferir na formação do vínculo materno-filial e no desmame, sendo coletados quando os animais estavam na pastagem.

A temperatura retal (TR) foi obtida com um termômetro clínico digital inserido no reto do animal, a uma profundidade de $3,5 \mathrm{~cm}$ e mantido por $3 \mathrm{~min}$; foram realizadas quatro coletas no terço final da gestação; uma coleta ao parto para ovelhas e cordeiros mensurados após a primeira mamada para não haver interferência nas observações comportamentais; e uma coleta no desmame para ovelhas e cordeiros.

A frequência respiratória (FR) foi medida contando-se o número de movimentos respiratórios no flanco dos animais por um período de 10 segundos e multiplicando-se os valores encontrados por 6 para se obter o número de movimentos respiratórios por minuto $\left(\right.$ mov.min $\left.{ }^{-1}\right)$; realizaram-se quatro coletas no terço final da gestação, uma coleta ao parto para ovelhas e cordeiros, mensuradas após a primeira mamada para não haver interferência nas observações comportamentais e uma coleta no desmame pata ovelhas e cordeiros.
A frequência cardíaca (FC) foi obtida por meio da contagem dos batimentos cardíacos, com auxílio de um estetoscópio e de um cronômetro por um período de 10 segundos, sendo o resultado multiplicado por 6 para se obter as batidas por minuto; foram realizadas quatro coletas no terço final da gestação; uma coleta ao parto, mensuradas após a primeira mamada para não haver interferência na formação do vínculo materno-filial para ovelhas e cordeiros; e uma coleta ao desmame para ovelhas e cordeiros, realizada em área de pastagem.

$\mathrm{O}$ índice de conforto térmico (ICT) foi desenvolvido especificamente para ovinos visando à classificação de ambientes para criação de ovinos, estimado por Barbosa \& Silva (1995), considerando a radiação e o vento como fatores importantes para estes animais. $\mathrm{O}$ ICT foi calculado pela seguinte fórmula: ICT $=0,6678 \mathrm{ta}+0,4969 \mathrm{p}+0,5444 \mathrm{tg}-$ $0,1038 \mathrm{v}$, em que ta $=$ temperatura do ar $\left({ }^{\circ} \mathrm{C}\right), \mathrm{p}=$ pressão parcial de vapor $(\mathrm{kPa})$, $\operatorname{tg}=$ temperatura do globo de Vernon, $\left({ }^{\circ} \mathrm{C}\right), \mathrm{v}=$ velocidade do vento $\left(\mathrm{m} \cdot \mathrm{s}^{-1}\right)$ (BARBOSA \& SILVA, 1995). Estes dados foram obtidos a partir do último mês de gestação, ao parto e ao desmame.

O delineamento estatístico utilizado foi o inteiramente casualizado, com duas repetições, utilizando-se o modelo estatístico SAS (STATISTICAL ANALYSIS SYSTEMS, 2009). Utilizouse o teste de Tukey em nível de 5\% de 
Rev. Bras. Saúde Prod. Anim., Salvador, v.16, n.1, p.199-209 jan./mar., 2015 http://www.rbspa.ufba.br ISSN 15199940

significância para comparação das médias.

\section{RESULTADOS E DISCUSSÃO}

Os critérios de tolerância e adaptação dos animais foram determinados pelas medidas fisiológicas da temperatura retal, frequência respiratória, frequência cardíaca e dos parâmetros ambientais por meio do índice de conforto térmico (ICT).

A temperatura retal das ovelhas no terço final de gestação sofreu influência climática, sendo maior $(\mathrm{P}<0,05)$ durante o verão do que no inverno (Tabela 2 ). $\mathrm{O}$ alto valor de TR durante o verão pode ter sido influenciado pelo ICT de 46 no período observado. $\mathrm{O}$ aumento na TR significa que o animal está estocando calor e ocorre o estresse calórico não havendo dissipação.

Tabela 2. Médias e desvios-padrão da temperatura retal (TR), frequência respiratória (FR), frequência cardíaca (FC) e índice de conforto térmico (ICT) no terço final de gestação, ao parto e no desmame, de ovelhas da raça Santa Inês no verão e no inverno

\begin{tabular}{|c|c|c|}
\hline \multirow{2}{*}{ Variável } & \multicolumn{2}{|c|}{ Terço final da gestação } \\
\hline & Verão & Inverno \\
\hline $\mathrm{TR}\left({ }^{\circ} \mathrm{C}\right)$ & $40,63 \pm 7,92^{\mathrm{a}}$ & $38,34 \pm 3,08^{b}$ \\
\hline $\mathrm{FR}\left(\right.$ mov. $\left.\mathrm{min}^{-1}\right)$ & $102,58 \pm 0,21^{\mathrm{a}}$ & $35,71 \pm 0,40^{\mathrm{b}}$ \\
\hline FC (bat.min $\left.{ }^{-1}\right)$ & $128,34 \pm 5,60^{\mathrm{a}}$ & $77,96 \pm 2,33^{\mathrm{b}}$ \\
\hline ICT & 46 & 14 \\
\hline \multicolumn{3}{|l|}{ Ao parto } \\
\hline TR $\left({ }^{\circ} \mathrm{C}\right)$ & $39,09 \pm 6,05^{\mathrm{a}}$ & $37,67 \pm 3,99^{\mathrm{b}}$ \\
\hline FR $\left(\operatorname{mov} \cdot \min ^{-1}\right)$ & $73,42 \pm 0,09^{\mathrm{a}}$ & $23,22 \pm 0,25^{\mathrm{b}}$ \\
\hline FC (bat.min $\left.{ }^{-1}\right)$ & $108,82 \pm 6,92^{\mathrm{a}}$ & $70,87 \pm 4,26^{\mathrm{b}}$ \\
\hline ICT & 37 & 17 \\
\hline \multicolumn{3}{|l|}{ Desmame } \\
\hline TR $\left({ }^{\circ} \mathrm{C}\right)$ & $39,62 \pm 6,05^{\mathrm{a}}$ & $39,07 \pm 3,99^{\mathrm{a}}$ \\
\hline $\mathrm{FR}\left(\right.$ mov. $\left.\min ^{-1}\right)$ & $87,97 \pm 0,09^{\mathrm{a}}$ & $28,20 \pm 0,25^{\mathrm{b}}$ \\
\hline FC (bat.min $\left.{ }^{-1}\right)$ & $123,52 \pm 6,92^{\mathrm{a}}$ & $77,77 \pm 4,26^{\mathrm{b}}$ \\
\hline ICT & 45 & 22 \\
\hline
\end{tabular}

Médias seguidas de letras diferentes diferem entre si pelo teste Tukey a 5\%.

Ao observar os parâmetros fisiológicos das ovelhas no terço final da gestação, verifica-se que no verão apresentou-se o maior ICT e no inverno o menor ICT, os resultados (Tabela 2), mostraram que no verão a TR ultrapassou os limites considerados normais para pequenos ruminantes. No entanto o ICT para o inverno não alterou a TR das ovelhas, mantendo-as dentro dos limites da zona de conforto térmico. Barbosa et al. (2001) observaram que no estado do
Paraná a TR aumentou para 40,4; 40,5 e $40,3^{\circ} \mathrm{C}$ para as raças Hampshire Down, Texel e Ile de France respectivamente quando o ICT atingiu 58.

A TR se manteve normal durante o verão quando o ICT foi de 37 , ao passo que no inverno com ICT 17 houve uma queda significativa na temperatura retal das ovelhas, já que o índice de conforto térmico apresentou-se divergente para ambas as estações. A queda na TR ao parto durante o inverno pode ter sido 
influenciado pela baixa condição corporal $(1,95 \pm 0,16)$ das ovelhas nesse período, pois foi observado que nesta estação as ovelhas apresentavam pêlos eriçados e tremores. No entanto, ovelhas que pariram no verão não apresentaram esse tipo de comportamento.

Ao avaliar a TR das ovelhas ao desmame, pôde-se constatar que este parâmetro manteve-se dentro dos níveis aceitáveis para pequenos ruminantes. Amaral et al. (2009) verificaram em raças ovinas produzidas no Paraná, incluindo a Santa Inês, valores de $39,17^{\circ} \mathrm{C}$ e $38,96^{\circ} \mathrm{C}$, respectivamente, para verão e inverno com temperaturas médias de $32,6^{\circ} \mathrm{C}$ e $12,55{ }^{\circ} \mathrm{C}$ respectivamente.Ao observar animais de raças de origem exóticas Barbosa \& Silva (1995) verificaram que a TR aumentou á partir de um ICT de 20 para ovinos da raça Sulfoolk e Corriedale e a raça Ideal manteve-se em homeotermia até o ICT de 35. Os resultados observados neste trabalho mostraram que para ovelhas nativas da raça Santa Inês durante o verão só aumentaram á partir do ICT 45 e no inverno diminuiu consideravelmente com ICT abaixo de 17. Pode-se afirmar que o ICT ideal para raça Santa Inês está entre 22 e 37 quando a temperatura do ar apresenta-se entre $16,9^{\circ} \mathrm{C}$ e $28,4^{\circ} \mathrm{C}$.

A taxa de respiração pode quantificar a severidade do estresse pelo calor em ruminantes, em que uma FR de 40 - 60, 60 - 80 e 80 - 120mov.min ${ }^{-1}$ caracterizam, respectivamente, estresse baixo, médioalto e alto e acima de 200 mov.min ${ }^{-1}$ o estresse seria classificado como severo para ovinos (SELANOKOVE, 2000).

Notou-se que a FR foi mais elevada durante o verão para todas as fases reprodutivas das ovelhas. No entanto, este fator fisiológico não se elevou durante o inverno e se manteve dentro dos limites considerados normais para pequenos ruminantes, sendo suficiente para manter a homeotermia, podendo afirmar que em nenhum dos animais estudados foram observados condições de estresse severo.

A FR, no terço final de gestação, sofreu alterações importantes, ocasionando aumento significativo para ovelhas avaliadas no verão (Tabela 2). Já no inverno, mostrou-se em níveis normais, revelando que as ovelhas da raça Santa Inês apresentam-se bem adaptadas à temperatura de $10,8^{\circ} \mathrm{C}$. Neiva et al. (2004), ao avaliarem o efeito do estresse climático sobre os parâmetros produtivos e fisiológicos de ovinos Santa Inês, observaram que a elevação da temperatura ambiente para $30,4^{\circ} \mathrm{C}$ no turno da tarde exerceu influência sobre a FR.

A FR ao parto foi maior $(\mathrm{P}<0,05)$ para ovelhas paridas no verão. Para as ovelhas paridas no inverno, a FR manteve-se dentro dos limites aceitáveis como normais para pequenos ruminantes $\left(20\right.$ a 50 mov. $\left.\mathrm{min}^{-1}\right)$ e durante o inverno a FR apresentou-se acima da normalidade com ICT 37 por ser o primeiro parâmetro fisiológico alterado quando a temperatura do ar aumenta, este mecanismo é utilizado para que o animal mantenha-se em conforto térmico. Entretanto, é preciso considerar que as condições climáticas (Tabela 1) apresentou-se com grande severidade durante o inverno, fator que não influenciou a mudança de comportamento da frequência respiratória, visto que o ICT foi de 17 ao parto. Neves et al. (2009) observaram valores críticos de FR quando ovelhas Santa Inês com pelame preto foram submetidas à ICT acima de 38, confirmando que a FR é o melhor parâmetro fisiológico indicador de estresse térmico para ovinos dessa raça. Para o período de desmame, foram encontradas diferenças significativas 
$(\mathrm{P}<0,05)$ entre as estações do ano verão e inverno, sendo que no verão a FR apresentou-se acima dos níveis considerados normais, enquanto no inverno não foi necessária a utilização deste parâmetro para dissipação de calor, visto que o ICT apresentou-se abaixo do encontrado no verão. Vale ressaltar que o mecanismo de perda de calor mais eficaz é o evaporativo, por não depender do diferencial de temperatura entre o organismo e a atmosfera. A evaporação respiratória é um mecanismo fisiológico utilizado pelos ovinos em respostas intensas por períodos mais curtos do dia (SILVA \& STARLING, 2003) o que corrobora com os resultados desta pesquisa, onde pode-se observar que durante o verão com ICT de 45 ovelhas utilizaram bem este mecanismo de troca de calor com o ambiente, visto que a TR manteve-se dentro dos limites aceitáveis.

A FC no terço final de gestação foi superior no verão e ultrapassou os níveis considerados normais para pequenos ruminantes sendo classificado como estresse alto, enquanto que no inverno os valores mantiveram-se dentro dos limites considerados normais (Tabela 2).

Os valores normais para FC está entre o intervalo de 70 e 80 bat.min ${ }^{-1}$. A FC das ovelhas ao parto manteve o mesmo comportamento do terço final da gestação no verão e no inverno. Os valores encontrados no verão são considerados altos quando comparadas com ovelhas nas mesmas condições ambientais e sem a presença de cordeiros. No entanto, durante o inverno, os batimentos cardíacos mostraram-se dentro do intervalo recomendado pela literatura para todos os estágios produtivos. Cardoso (2008) encontrou valores de 76,54bat.min ${ }^{-1}$ no verão/seco e 59,25 bat.min ${ }^{-1}$ no inverno/seco. Da mesma forma, durante o desmame, a FC no verão apresentou-se acima das médias consideradas normais. No entanto, Cezar (2004) encontrou valores de 96,50bat.min ${ }^{-1}$ e 99,98bat.min ${ }^{-1}$ para o inverno e o verão, respectivamente, na região semiárida do Nordeste do Brasil onde a temperatura mínima encontrada foi de $23,3^{\circ} \mathrm{C}$, contrário a condição de realização desta pesquisa que apresentou no inverno temperatura de $10,8^{\circ} \mathrm{C}$.

Em relação aos parâmetros fisiológicos para cordeiros do nascimento ao desmame observa-se que a TR e FR mantiveram-se dentro dos limites aceitáveis, enquanto a FC mostrou-se acima dos limites considerados normais no verão e inverno (Tabela 3 ).

Os ICT, ao nascer e ao desmame, foram superiores para cordeiros nascidos no verão. Ao nascimento, a TR e FR apresentaram-se superiores no verão $(\mathrm{P}<0,05)$ quando comparados aos nascimentos ocorridos no inverno (Tabela 3). A TR e FR, embora diferentes, encontram-se dentro dos padrões normais para pequenos ruminantes. Ao nascimento, os cordeiros possuem pouco tecido adiposo marrom e por encontrar o meio diferente do ambiente intrauterino, a temperatura corporal pode sofrer variações de $1^{\circ} \mathrm{C}$ a $2^{\circ} \mathrm{C}$ neste período, situação idêntica ocorreu durante $\mathrm{o}$ inverno. No entanto, a FC dos cordeiros nascidos no inverno foi superior aos nascidos no verão, certamente pelo fato de aumentar a circulação sanguínea para se manterem aquecidos.

No desmame, não houve efeitos entre as estações verão e inverno para TR $(\mathrm{P}>0,05)$; porém a FR e FC $(\mathrm{P}<0,05)$, apresentaram efeito. As altas taxas respiratórias observadas durante $\mathrm{o}$ inverno ao desmame podem ter sido superiores pelo manejo utilizado no momento da aferição, ao passo que os cordeiros se movimentavam pelo pasto por estarem independentes no momento da aferição. 
Rev. Bras. Saúde Prod. Anim., Salvador, v.16, n.1, p.199-209 jan./mar., 2015 http://www.rbspa.ufba.br ISSN 15199940

Tabela 3. Médias e desvios-padrão para os parâmetros fisiológicos de cordeiros 1/2 Dorper - Santa Inês ao nascimento e desmame, no verão e inverno

\begin{tabular}{|c|c|c|c|}
\hline \multirow{2}{*}{ Variáveis } & \multicolumn{2}{|c|}{ Nascimento } & \multirow{2}{*}{$\mathrm{CV}(\%)$} \\
\hline & Verão & Inverno & \\
\hline $\mathrm{TR}\left({ }^{\circ} \mathrm{C}\right)$ & $39,42 \pm 0,28^{\mathrm{a}}$ & $38,22 \pm 0,42^{\mathrm{b}}$ & 0,93 \\
\hline FR (mov.min $\left.{ }^{-1}\right)$ & $74,86 \pm 7,19^{\mathrm{a}}$ & $65,69 \pm 7,76^{\mathrm{b}}$ & 6,25 \\
\hline FC (bat.min $\left.{ }^{-1}\right)$ & $188,08 \pm 5,36^{\mathrm{b}}$ & $216,14 \pm 4,54^{\mathrm{a}}$ & 2,39 \\
\hline ICT & 37 & 17 & - \\
\hline \multicolumn{4}{|c|}{ Desmame } \\
\hline $\mathrm{TR}\left({ }^{\circ} \mathrm{C}\right)$ & $39,10 \pm 4,31^{\mathrm{a}}$ & $39,07 \pm 6,97^{\mathrm{a}}$ & 0,27 \\
\hline $\mathrm{FR}\left(\mathrm{mov} \cdot \mathrm{min}^{-1}\right)$ & $75,84 \pm 0,12^{\mathrm{b}}$ & $114,25 \pm 0,08^{\mathrm{a}}$ & 6,58 \\
\hline FC (bat.min $\left.{ }^{-1}\right)$ & $119,73 \pm 2,10^{\mathrm{a}}$ & $96,76 \pm 1,86^{\mathrm{b}}$ & 3,13 \\
\hline ICT & 45 & 22 & - \\
\hline
\end{tabular}

Médias seguidas de letras diferentes, diferem entre si pelo teste de Tukey a 5\%.

$\mathrm{TR}=$ temperatura retal, $\mathrm{FR}=$ frequência respiratória, $\mathrm{FC}=$ frequência cardíaca $\mathrm{e} \mathrm{ICT}=$ índice de conforto térmico.

As variáveis $\mathrm{FR}$ e $\mathrm{FC}$, no verão e no inverno dos cordeiros no desmame (Tabela 3). Moraes (2011), avaliando ovelhas da raça Corriedale, encontrou FR ao desmame dos cordeiros de 63,38mov.min ${ }^{-1}$ e FC de 106,13bat.min ${ }^{-1}$. A FC dos cordeiros ao desmame apresentou-se maior no verão do que no inverno, valores estes superiores aos considerados normais com valores de 70 e 80 bat. $\mathrm{min}^{-1}$. Isto se deve ao fato de os cordeiros apresentarem-se desenvolvidos aos 60 dias no momento do desmame e as aferições fisiológicas terem sido realizadas ao ar livre, contribuindo com o aumento na FC, pois os cordeiros se movimentavam em situação de fuga quando presos para as observações o que não ocorreu quando as aferições foram realizados ao nascimento.

O escore de condição corporal ao parto das ovelhas apresentou-se superior $(\mathrm{P}=0,0001)$ durante o verão, bem como o desempenho produtivo dos cordeiros nascidos no verão foi superior aos dos nascidos no inverno (Tabela 4).

Os valores do ECCP foram de 2,93 e 1,95 , respectivamente, para o verão e o inverno, o que evidencia que apenas ovelhas paridas no verão estavam em condições adequadas para se reproduzirem, indicando necessidade de manejo diferenciado para as ovelhas em ambas as estações.

Tabela 4. Médias e desvios-padrão do escore da condição corporal das ovelhas ao parto (ECCP), peso ao nascer (PN), peso a desmama (PD), ganho de peso diário (GPD) e taxa de mortalidade dos cordeiros

\begin{tabular}{lccc}
\hline Variáveis & Verão & Inverno & CV $(\%)$ \\
\hline ECCP & $2,93 \pm 0,26^{\mathrm{a}}$ & $1,95 \pm 0,16^{\mathrm{b}}$ & 8,76 \\
PN $(\mathrm{kg})$ & $4,51 \pm 2,86^{\mathrm{a}}$ & $3,57 \pm 2,31^{\mathrm{b}}$ & 70,44 \\
PD $(\mathrm{kg})$ & $16,93 \pm 3,91^{\mathrm{a}}$ & $14,32 \pm 0,67^{\mathrm{b}}$ & 16,56 \\
GPD (g/dia) & $0,207 \pm 0,07^{\mathrm{a}}$ & $0,179 \pm 0,03^{\mathrm{b}}$ & 29,00 \\
\hline Mortalidade (\%) & 4,6 & 16,49 & - \\
\hline
\end{tabular}

Médias seguidas de letras diferentes diferem entre si pelo teste de Tukey a 5\%.

$\mathrm{CV}=$ coeficiente de variação. 
Houve diferença significativa do $\mathrm{PN}$ $(\mathrm{P}<0,05)$ para os cordeiros nascidos no verão e no inverno. Esta diferença se deve à condição corporal da ovelha durante a gestação, visto que ovelhas que pariram no verão tiveram acesso à pastagem com melhor qualidade do que as ovelhas que pariram no inverno. Médias de peso ao nascer de $4,82 \mathrm{~kg}$ foram encontradas por Santello et al. (2010) para cordeiros $1 / 2$ Dorper - Santa Inês nascidos no verão, pesos superiores ao deste trabalho, provavelmente, pela suplementação no início da gestação.

Durante o terço final da gestação ocorre em torno de $90 \%$ do desenvolvimento do feto e de seus envoltórios, o não atendimento das exigências nutricionais das matrizes acarreta diminuição de até $40 \%$ do peso total no desenvolvimento fetal (CUNHA et al., 2005).

$\mathrm{O}$ estresse térmico influencia o final da gestação. Em particular na ovelha, a exposição a temperaturas elevadas durante o último terço da gestação retarda o crescimento fetal, efeitos esses que também são verificados em vacas e cabras (McCRABB et al., 1993). No entanto, estas observações não se confirmaram para ovelhas da raça Santa, ao passo que os cordeiros nascidos no verão com ICT de 45 no terço final de gestação apresentaram pesos compatíveis a raça (Tabela 3). Ao nascimento, obtiveram-se cordeiros com melhor $(\mathrm{P}<0,05)$ PN em relação aos cordeiros provenientes de ovelhas mantidas em ambientes com ICT menor (17) durante o inverno.

Cordeiros com baixo peso ao nascer apresentam pouco tecido de reserva corporal, são menos vigorosos, possuem menor temperatura corporal e levam mais tempo para levantar e encontrar o úbere (DWYER et al., 2005).

O GPD de cordeiros nascidos durante o verão foi significativamente superior $(\mathrm{P}<0,05)$ ao observado no inverno, proporcionando também desmame de cordeiros com 60 dias, com peso médio superior. Santello et al. (2010) verificaram o ganho de peso diário de cordeiros 1/2 Dorper - Santa Inês nascidos no verão e provenientes de ovelhas suplementadas no início da gestação e observaram médias de $0,179 \mathrm{~kg} /$ dia.

A condição corporal ao parto tem grande influência na produção de cordeiros proporcionando superioridade para o peso ao nascer, peso no desmame e ganho de peso diário.

Cordeiros, filhos de ovelhas com ECCP entre 2,5 e 3,0, foram significativamente superiores $(\mathrm{P}<0,05)$ para $\mathrm{PN}$ e GPD quando comparados aos das ovelhas com ECCP entre 1,5 e 2,0 (Tabela 5).

Tabela 5. Médias do peso ao nascer (PN), peso no desmame (PD) e ganho de peso diário (GPD) dos cordeiros em função da condição corporal das ovelhas (CC)

\begin{tabular}{cccc}
\hline \multirow{2}{*}{ Variável } & \multicolumn{3}{c}{ Escore de Condição Corporal } \\
\cline { 2 - 4 } & $(1,5-2,0)$ & $(2,0-2,5)$ & $(2,5-3,0)$ \\
\hline PN & $3,50^{\mathrm{b}}$ & $3,92^{\mathrm{b}}$ & $4,60^{\mathrm{a}}$ \\
PD & $14,40^{\mathrm{b}}$ & $16,36^{\mathrm{b}}$ & $19,20^{\mathrm{a}}$ \\
GPD & $0,181^{\mathrm{b}}$ & $0,207^{\mathrm{b}}$ & $0,242^{\mathrm{a}}$ \\
\hline
\end{tabular}

Médias seguidas de letras diferentes, nas linhas diferem-se entre si $(\mathrm{P}<0,05)$ pelo teste de Tukey a $5 \%$.

Greenwood et al. (2002) observaram que cordeiros Suffolk x Finnsheep-Dorset nascidos com baixo peso $(2,28 \mathrm{~kg})$, obtiveram ganho de peso diário de $0,150 \mathrm{~kg}$, enquanto cordeiros com alto 
peso ao nascer $(4,84 \mathrm{~kg})$ alcançaram ganhos médios de $0,337 \mathrm{~kg}$.

Um fator importante para produção de cordeiros é o peso do cordeiro ao nascer e ao desmame, pois são altamente dependentes da condição corporal da ovelha durante a gestação e no período de aleitamento. Para que seja aproveitado todo potencial de ganho de peso até o desmame, segundo Geraseevt et al. (2006), a restrição nutricional na vida uterina pode comprometer a produtividade de cordeiros após o desmame, mesmo com aleitamento em abundância durante a fase de dependência materna, reflexo do baixo peso ao nascimento.

Conclui- se que ovelhas da raça Santa Inês estão adaptadas às condições climáticas nas estações verão e inverno com temperaturas médias que variam de $10,8^{\circ} \mathrm{C}$ a $33,5^{\circ} \mathrm{C}$. Nas condições de realização do experimento, ovelhas paridas quando a temperatura média foi de $28,4^{\circ} \mathrm{C}$ demonstraram produção de cordeiros com melhor desempenho. Porém há de se considerar que a produção de cordeiros pode ser beneficiada com adoção de estratégias para minimizar os efeitos ambientais durante o inverno sobre o sistema de produção

\section{REFERÊNCIAS}

AMARAL, F.F.; BARBOSA, O.R.; GASPARINO, E. AKIMOTO, L.S.; LOURENÇO, F.J.; SANTELLO, G.A. Efeito da suplementação alimentar nas respostas fisiológicas, hormonais e sanguíneas de ovelhas Santa Inês, Ile de France e Texel. Acta Scientiarum

Animal Sciences, v.31, n.4, p.403-410, 2009.
BARBOSA, O.R.; MACEDO, F.A.F.; GROES, R.V.G.; GUEDES, J.M.F. Zoneamento bioclimático da ovinocultura do Estado do Paraná. Revista Brasileira de Zootecnia, v.30, n.2, p.454-460, 2001.

BARBOSA, O.R.; SILVA, R.G. Índice de conforto térmico para ovinos. Boletim de Indústria Animal, v.52, n.1, p. 29-35, 1995.

BLACKSHAW, J.K. Noteson some topics in applied animal behavior. Queensland: University of Queensland, 2003.

CARDOSO, F.S. Termoregulação de ovinos da raça Santa Inês e da raça Dorper no meio-norte do Brasil. 2008. 33f. Dissertação (Mestrado em Ciência Animal) - Universidade Federal do Piauí, Teresina.

CEZAR, M.F. Avaliação de parâmetros fisiológicos de ovinos Dorper, Santa Inês e seus mestiços perante condições climáticas do tropical semi-árido nordestino. Ciência e Agrotecnologia, v.28, n.3. p.614-620, 2004.

CORNER, R.A.; KENYON, P.R.; STAFFORD, J.K..; WEST, D.M.; OLIVER, M.H. The effect of midpregnancy shearing or yarding stress on ewe post-natal behavior of their lambs. Livestock Science, v.102, n.1-2, p.121129, 2006.

CUNHA, E.A.; SANTOS, L.E.; BUENO, M.S.Cordeiros para abate super precoce. São Paulo: APTA/SAA, 2005.

DWYER, C.M.; LAWRENCE, A.B. A review of the behavioural and physiological adaptations of hill and lowland breeds of sheep that favour lamb survival. Applied Animal Behaviour Science, v.92, n.3, p.235-260, 2005. 
Rev. Bras. Saúde Prod. Anim., Salvador, v.16, n.1, p.199-209 jan./mar., 2015 http://www.rbspa.ufba.br ISSN 15199940

GERASEEVT, L.C.; PEREZ, J.R.O.; CARVALHO, P.A. Efeitos das restrições pré e pós-natal sobre o crescimento e o desempenho de cordeiros Santa Inês do nascimento ao desmame. Revista Brasileira de Zootecnia, v.35, n.1, p.245-251, 2006.

GREENWOOD, P.L.; HUNT, A.S.; SLEPETIS, R.M. ; FINNERTY, K.D.; ALSTON, C.; BEERMANN, D.H.; BELL, A.W. Effects of birth weight and postnatal nutrition on neonatal sheep: III. Regulation of energy metabolism.

Journal of Animal Science, v.80, n.11, p.2850-2861, 2002.

McCRABB, G.J.; McDONALD, B.J.; HENNOSTE, L.M. Heat stress during mid-pregnancy in sheep and the consequences for placental and fetal growth. Journal Agriculture Science, v.120, n.2, p.265-271, 1993.

MORAES, A.B. Habilidade materna de ovelhas Corriedale e sua relação com a sobrevivência e desenvolvimento de cordeiros. 2011. 99f. Dissertação (Mestrado em Zootecnia) - Universidade Federal do Rio Grande do Sul, Porto Alegre.

NEIVA, J.N.M.; TEXEIRA, M.; TURCO, S.H. OLIVEIRA, S.M.P.; MOURA, A.A.A.N. Efeito do estresse climático sobre os parâmetros produtivos e fisiológicos de ovinos Santa Inês mantidos em confinamento na região Litorânea do Nordeste do Brasil. Revista Brasileira de Zootecnia, v.33, n.3, p.668-678, 2004.

NEVES, M.L.M.W.; AZEVEDO, M.; COSTA, L.A.B.; GUIN, A.; LEITE, A.M.; CHAGAS, J.C. Níveis críticos do índice de conforto térmico para ovinos da raça Santa Inês criados a pasto no agreste do estado do Pernambuco. Acta

Scientiarum. Animal sciences, v.31, n.2, p.169-175, 2009.
NOWAK, R.; POINDRON, P. From birth to colostrum: early steps leading to lamb survival. Reprodution Nutrition Development, v.46, n.4, p.431-446, 2006.

RUCKEBUSCH, Y.; PHANEAUF, L.F.; DUNLOP, R. Physiology of small and large animals. Philadelphia: Decker, 1991. p.399-406.

SANTELLO, G.A.; MACEDO, F.A.F.; LOURENÇO, F.J. Morfologia muscular e características qualitativas da carne de cordeiros $1 / 2$ Dorper - Santa Inês.

Revista Brasileira da Saúde e Produção Animal [online], v.11, n.3, p.876-887, 2010.

SELANIKOVE, N. Effects of heat stress on the welfare of extensively managed domestic ruminants.

Livestock Production Science, v.67, n.1, p.1-18, 2000. SILVA, R.G. Introdução à bioclimatologia animal. São Paulo: Nobel, 2000. 286p.

SILVA, R.G.; STARLING, J.M.C. Evaporação cutânea e respiratória em ovinos sob altas temperaturas ambientes. Revista Brasileira de Zootecnia, v.32, n.6, p.1956-1961, 2003. Supl. 2.

\section{STATISTICAL ANALYSIS} SYSTEMS. SAS/STAT user's guide: statistics. Cary, NC: SAS Institute, 2009.

Data de recebimento: 02/12/2013

Data de aprovação: 06/03/2015 